\title{
What genomic data can reveal about eco-evolutionary dynamics
}

Seth M. Rudman*1, Matt A. Barbour², Katalin Csillery³, Phillip Gienapp ${ }^{4}$, Frederic Guillaume ${ }^{2}$, Nelson G. Hairston Jr. ${ }^{5}$, Andrew P. Hendry ${ }^{6}$, Jesse R. Lasky ${ }^{7}$, Marina Rafajlović ${ }^{8,9}$, Katja Räsänen ${ }^{10}$, Paul S. Schmidt ${ }^{1}$, Ole Seehausen ${ }^{11,12}$, Nina 0. Therkildsen $^{13}$, Martin M. Turcotte ${ }^{3,14}$, and Jonathan M. Levine ${ }^{15}$

1 Department of Biology, University of Pennsylvania, Philadelphia, Pennsylvania, United States of America

2 Department of Evolutionary Biology and Environmental Studies, University of Zurich, Zurich, Switzerland

${ }^{3}$ ETH Zürich, Adaptation to a Changing Environment, Zurich, Switzerland

4 Netherlands Institute of Ecology (NIOO-KNAW), Department of Animal Ecology, Wageningen, the Netherlands

${ }^{5}$ Department of Ecology and Evolutionary Biology, Cornell University, Ithaca, New York, United States of America

${ }^{6}$ Redpath Museum and Department of Biology, McGill University, Montreal, Quebec, Canada

7 Department of Biology, Pennsylvania State University, University Park, Pennsylvania, United States of America

8 Department of Physics, University of Gothenburg, Gothenburg, Sweden

${ }^{9}$ Centre for Marine Evolutionary Biology, University of Gothenburg, Tjärnö, Strömstad, Sweden

10 Eawag, Dept. of Aquatic Ecology/ETH-Zurich, Institute of Integrative Biology, Switzerland

${ }^{11}$ Department of Fish Ecology and Evolution, EAWAG Swiss Federal Institute of Aquatic Science and Technology, Center for Ecology, Evolution and Biogeochemistry, Kastanienbaum, Switzerland

${ }^{12}$ Aquatic Ecology and Evolution, Institute of Ecology and Evolution, University of Bern, Bern, Switzerland

13 Department of Natural Resources, Cornell University, Ithaca, New York, United States of America

14 Department of Biological Sciences, University of Pittsburgh, Pittsburgh, USA

15 Institute of Integrative Biology, ETH Zürich, Zürich, Switzerland

${ }^{*}$ Corresponding author 


\begin{abstract}
Recognition that evolution operates on the same timescale as ecological processes has motivated growing interest in eco-evolutionary dynamics. Nonetheless, generating sufficient data to test predictions about eco-evolutionary dynamics has proven challenging, particularly in natural contexts. Here we argue that genomic data can be integrated into the study of eco-evolutionary dynamics in ways that deepen our understanding of the interplay between ecology and evolution. Specifically, we outline five major questions in the study of eco-evolutionary dynamics for which genomic data may provide answers. Although genomic data alone will not be sufficient to resolve these challenges, integrating genomic data can provide a more mechanistic understanding of the causes of phenotypic change, help elucidate the mechanisms driving eco-evolutionary dynamics, and lead to more accurate evolutionary predictions of eco-evolutionary dynamics in nature.
\end{abstract}


Evidence that the ways organisms interact with their environment can evolve fast enough to alter ecological dynamics has forged a new link between ecology and evolution ${ }^{1-5}$. A growing area of study, termed eco-evolutionary dynamics, centers on understanding when rapid evolutionary change is a meaningful driver of ecological dynamics in natural ecosystems ${ }^{6-8}$. Among the most interesting of these dynamics are eco-evolutionary feedbacks, where evolution alters ecological processes and this then shapes the course of subsequent evolution 9,10. Empirically evaluating the prevalence and importance of such feedbacks in nature is challenging but see 3,10 , but doing so has the potential to provide a more comprehensive and mechanistic understanding of the relationships between ecological processes and the mechanisms driving rapid evolution $7,8,11$. More generally, better resolving eco-evolutionary dynamics has great potential to improve our understanding of processes ranging from community assembly to ecological speciation and adaptive radiation. Nonetheless, the number of comprehensive case studies of eco-evolutionary dynamics is modest and new tools are needed to explore these dynamics in a wider range of ecosystems, as well as to strengthen inference from existing work.

In recent years, the study of rapid evolution, one component of the eco-evolutionary dynamic, has benefitted from the increasing availability of genomic sequence data ${ }^{12-14}$. Genomic data have been used, for example, to demonstrate that while selection on individual loci can be strong ${ }^{15}$, rapid adaptation often occurs through selection on many loci 1,16 . This information has great potential utility for understanding eco-evolutionary dynamics because rapid trait evolution plays a central role in regulating such dynamics. Particularly relevant is information gleaned from efforts to understand the genomic basis of adaptation, a burgeoning field in evolutionary biology that investigates the specific genomic underpinnings of phenotypic variation, its response to natural selection, and effects on fitness ${ }^{17}$. Hence, while the genomics of eco-evolutionary dynamics is, in part, a specific application of broader efforts to understand the genomic basis of changes in functional traits 8,18 , the relevance of this body of work to answering key questions in the study of eco-evolutionary dynamics has yet to be fully articulated.

Here we identify five major questions in the study of eco-evolutionary dynamics, all of which remain largely unanswered, for which the incorporation of genomic data may facilitate progress (Fig. 1): (1) How often is evolution fast enough to drive ecological change? (2) How important are the effects of evolution on ecological dynamics relative to other ecological drivers? (3) Which phenotypes drive eco-evolutionary dynamics? (4) What is the genomic basis of phenotypes with large community- and ecosystem-level effects? (5) How repeatable are eco-evolutionary dynamics? In general, genomic data alone will not be sufficient to address these questions and much of the utility that comes from using genomic tools may currently only be feasible in genetic model systems. However, integrating these data into studies of eco-evolutionary dynamics can provide a better mechanistic understanding of the causes of phenotypic change, help elucidate the mechanisms driving eco-evolutionary dynamics, and lead to more accurate evolutionary predictions of eco-evolutionary dynamics in nature (Fig. 2). 


\section{How often is evolution fast enough to drive ecological change?}

Once thought too slow to affect ecological dynamics ${ }^{19}$, evolutionary change (i.e. heritable change in trait values or trait frequencies) has now been demonstrated to operate on a similar timescale as ecological processes in a large number of cases 2,5,20,21. Many of the existing studies in eco-evolutionary dynamics have assessed how genetic variation and divergence arising from evolution over decades to centuries alter ecological dynamics 22-25. Meanwhile, laboratory-based studies and a growing number of field experiments and a growing number of laboratory-based studies have demonstrated that evolution occurring over the course of an experiment can impact ecological dynamics. For example, rapid evolution of algal populations in response to differential selection by predatory rotifers can lengthen the population cycle period and shift the relative phasing of predator and prey ${ }^{1}$. Nonetheless, understanding when and how often evolution is fast enough to alter ecological dynamics in nature remains among the greatest uncertainties in the study of eco-evolutionary dynamics.

Until recently, studying evolution in natural populations was restricted to measuring temporal changes or spatial differences in phenotype, tracking the frequency of clones, or measuring changes in breeding values or allele frequencies at a few genetic markers. Measuring rapid evolution of phenotypes remains tremendously useful for the study of eco-evolutionary dynamics, because doing so simultaneously documents the pace of evolution and potentially relevant trait change. Yet, measures of evolution from phenotypic change have limitations. First this approach is typically limited to traits that are straightforward to measure (i.e. body size, coloration) ${ }^{26}$, meaning that potentially informative but difficult to measure phenotypes, such as those related to physiology or functional morphology, are less frequently chosen for studies on rapid evolution. Second, because a limited number of phenotypes are measured when studying rapid evolution, it is possible (and perhaps even likely) to entirely miss other rapidly evolving traits. Finally, trait shifts in natural environments could stem from plasticity, and thus disentangling the relative importance of environmentally induced and genetic responses requires the use of a common garden to confirm that observed trait shifts are heritable 27,28 . Though not without limitations, the incorporation of genomic data can help resolve each of these challenges.

Whole genome data and advances in bioinformatics now allow researchers to search for signatures of natural selection in the genomes of natural populations without a priori knowledge of links between genotype and phenotype. Possible signatures of selection include reduced genetic diversity in the area of the genome where an allele is under selection ${ }^{29}$, distinct patterns in haplotype structure and linkage disequilibrium ${ }^{30,31}$, as well as variation in allele frequencies along environmental gradients. Direct measurements of allele frequency changes over time have also shown that shifts can occur stunningly fast when standing variation is present 16,32 . Compared to the tools 
available just a decade ago, our ability to now use genome-wide data to look for evidence of allele frequency changes expands the scope for detecting rapid evolution because the number of variants measured is tremendous. This could have particular utility in cases where eco-evolutionary dynamics are driven by cryptic phenotypic changes 33 . Finally, the simultaneous collection of genomic data from interacting species over time presents the opportunity to track how adaptive changes in one species relate to both ecological and adaptive changes in the other.

Understanding which evolutionary mechanism caused or facilitated the rapid evolutionary changes inferred from genomics data nonetheless remains challenging. Genetic drift, gene flow, hybridization, and genomic hitchhiking can produce patterns that may look like selection 15,34-37. Reliably detecting selection requires the use of population genetic models, simulations, and/or statistical analyses that consider the possibility that non-selective processes produced the observed patterns 15,29,34,38. Alternatively, using replicated experiments, be they natural or human made, to identify regions of the genome that show signatures of selection in multiple experimental replicates (a very conservative approach in part because the same regions of the genome may not show selection even when selection acts in parallel across replicates), provides a way to confidently detect selection. Finally, although these genomic methods can provide compelling evidence of a heritable response to selection, they are not direct evidence of phenotypic evolution. Quantifying such phenotypic change, as well as the ecological consequences of this evolution requires other approaches, some of which we will outline in the "Which phenotypes drive eco-evolutionary dynamics?" section.

\section{Box 1 here}

\section{How important are the effects of evolution on ecological dynamics relative to other ecological drivers?}

One of the major goals of research on eco-evolutionary dynamics is to understand the relative importance of rapid evolutionary change versus non-evolutionary ecological processes (e.g. rainfall) in driving population dynamics, community structure, and ecosystem processes ${ }^{39}$. At the most basic level, addressing this question requires simultaneously evaluating the ecological effects of genetic changes (at the population level) or differences (at the individual or population level) and comparing these effects to those of other ecological processes. Experiments that have assessed the effects of pre-existing genetic variation between members of diverged lineages have illustrated that adaptation can be a driver of ecological dynamics on par with traditionally explored processes such as predation or population density 24,40 . This can also be true for evolution occurring over short timescales. Turcotte et al. ${ }^{9}$ demonstrated that aphid evolution had a similar or stronger impact than a threefold change in initial population density on aphid population dynamics over the course of an experiment. 
However, experiments of this sort are labor intensive, so the use of observational data to infer the role of evolution in driving ecological dynamics ${ }^{2}$ is highly attractive. Hairston et al. ${ }^{2}$ developed a framework for partitioning the variance in population growth rate over time into contributions of ecological versus evolutionary drivers 41,42 . These evolutionary drivers reflect the influence on population size of temporal changes traits - beak size relative to seed availability in the Darwin's finch system, for example. However, to get a true estimate of the importance of evolution for ecological dynamics on the basis of this method it is crucial to ensure that the observed trait change is heritable and not simply plasticity, which can prove difficult for many species in nature 41,42. Genomics can be useful for resolving this difficulty.

With genomic data one can estimate the heritability of traits in a natural population without conducting labor intensive common garden experiments (Box 2). With nextgeneration sequencing it is now possible to estimate pairwise relatedness among individuals even in wild populations of non-model organisms ${ }^{43,44}$. This approach replaces the pedigree-based genetic relationship matrix of a quantitative genetic model with a genomic relationship matrix (GRM) estimated from genetic markers (see Box 2 for details). Estimates of the additive genetic variance (i.e. the part of the trait variance due to resemblance between relatives; the numerator in heritability) can provide a measure of evolutionary potential given that an appropriate scaling is used ${ }^{45}$. By coupling trait heritabilities with information on trait change and ecological changes through time it is possible to quantitatively assess the relative importance of rapid evolution in natural populations 41,46 .

Genomics could also be used to evaluate the temporal association between evolution in one species of an interacting pair and evolution in the other. More specifically, genomic sequence data collected through time could be used to track adaptive changes in allele frequency in two species that have a strong ecological interaction. Simultaneously measuring species' abundance and the strength of the interaction between them would allow one to test the association between ecological and evolutionary change in the two species. Although this approach would largely ignore phenotypes, the combined genomic and ecological dataset would allow one to quantify the association between evolutionary change in each of the two species that is specifically correlated with the strength of their interaction. To our knowledge this has not been attempted and it would be best first tried in an experimental setting, where the strength of the interaction between species could be manipulated or replicated, to provide stronger evidence that evolutionary change in both focal species stems from evolution in the other. This approach to studying evolution in real time while also collecting data on ecological dynamics could yield novel insight into the relative importance of rapid evolution in shaping ecological interactions. 


\section{Box 2 here}

\section{Which phenotypes drive eco-evolutionary dynamics?}

In order for eco-evolutionary dynamics to operate, phenotypes must evolve quickly and have sizeable ecological effects 7,11 . Lab-based rotifer-algal chemostat experiments have identified evolution in prey-defense phenotypes (e.g. algal clumping) ${ }^{47}$, yet many of the field-based eco-evolutionary dynamics experiments do not identify the specific phenotypes responsible for measured ecological effects (but see ${ }^{48,49}$ ). This lack of phenotypes in studies of eco-evolutionary dynamics, particularly field studies focused on the ecological effects of evolution, stems in part from a large number of possible relevant phenotypes. Even in cases where numerous phenotypes are measured, it is still difficult to be certain that the most crucial traits have been identified. Overcoming these limitations is important because traits determine the outcome of ecological interactions and ultimately shape communities and ecosystems ${ }^{50}$. Thus, developing a better understanding of the phenotypic basis of eco-evolutionary dynamics may help identify which ecological interactions are key agents of selection and help predict subsequent evolutionary change. Furthermore, more complete phenotypic information would illuminate whether eco-evolutionary dynamics are driven by evolution in a few or many traits.

Genomic data could be particularly useful for identifying the traits responsible for ecoevolutionary dynamics in genetic model organisms or closely related taxa. A sequenced genome makes it easier to identify genes under selection in an experimental manipulation or time series (as discussed in question 1). Yet, to relate this information to phenotypes, functional information must be available for the genes under selection. Gene function in model organisms can be investigated using compiled databases (e.g. Flybase 51, The Arabidopsis Information Resource ${ }^{52}$ ). Although the functional annotations may not account for the pleiotropic nature of many alleles, they would provide a starting point for exploration that could identify phenotypes that were not considered previously.

More manipulative approaches can also be used to identify the traits driving ecoevolutionary dynamics. With genetic lines that are fixed for a given allele at a previously identified locus, but vary across the rest of the genome, one can follow up on known annotations to explore the effects of an allele on previously identified phenotypes. Allelic replacement techniques (i.e. Clustered regularly interspaced short palindromic repeats (CRISPR) ${ }^{53}$, near isogenic lines (NILs) ${ }^{54}$, and transfer DNA (TDNA) 55 that potentially alter just a single locus within a consistent genomic background would streamline this process. These 'reverse phenotyping' approaches would be most tractable in model systems where producing inbred lines is feasible and where gene functions are more likely to be known. Using lines that have been altered by allelic replacement techniques for field experiments could be technologically challenging and 
certainly warrants careful ethical consideration. However, for those working in genetic model systems, genomic information could help identify phenotypes that drive ecoevolutionary dynamics 56.

\section{What is the genomic basis of phenotypes with large community and ecosystem level effects?}

As is known from numerous studies of the genomic basis of adaptation, the genotype-tophenotype map can be used to evaluate the nature and complexity of the genomic basis of traits in natural populations ${ }^{13,15}$. However, relatively few studies comprehensively explore the full genotype-to-phenotype-to-ecology relationship (Fig. 1) by investigating how genes under selection influence communities and ecosystems. In addition, there has been relatively little discussion of exactly how information gleaned from this relationship can inform eco-evolutionary dynamics. As such, we review the methodological developments in genotype-to-phenotype mapping and discuss their relevance to eco-evolutionary dynamics here.

Recent advances in understanding the genetic basis of phenotypic variation, including phenotypes that have large ecological effects ${ }^{57,58}$, have been made through the use of association mapping and quantitative trait locus (QTL) mapping (Box 3), which detect statistical associations between genotype and phenotype ${ }^{59}$. In addition to looking for loci correlated with variation in ecologically relevant traits, studies in ecological genomics have used the same tools to directly measure associations between genetic variation and community or ecosystem level variation (i.e. using community or ecosystem variation as a phenotype) ${ }^{60,61}$. The amount of information that can be gleaned from association based approaches depends in part on what other data is available. In cases where there is no linkage map or reference genome, association based approaches can provide basic information about the total number of markers associated with an ecologically relevant phenotype. When combined with a linkage map, the same association approaches could detail how many or what proportion of physically independent loci are associated with a given phenotype and their respective contribution to the total phenotypic variance explained. With an assembled, wellannotated and physically anchored genome, we can additionally obtain a list of candidate genes that may influence the phenotype of interest.

For investigations of eco-evolutionary dynamics, there is limited utility to lists of loci associated with particular phenotypes alone. An exception are cases where single genomic regions can have large effects on phenotypes and can lead to changes in ecological interactions and ecosystem functions 62,63. Using inbred lines or allelic replacement technologies, or directly sequencing and removing variation at these specific loci could allow for the explicit investigation of the importance of evolution from standing genetic variation at a single locus in driving eco-evolutionary dynamics. 
These experiments may be most tractable in well-studied genetic model systems, but they could provide a unique mechanistic view of eco-evolutionary dynamics in systems where variation in a single gene has large phenotypic and ecological effects. However, in the vast majority of cases ecologically important phenotypes will almost certainly be controlled by many genes ${ }^{64-66}$ and many phenotypes may drive ecological dynamics 64,67 . These considerations potentially reduce the value of identifying the effects of particular genetic variants, as the individual effects of a single locus on a phenotype (or extended phenotype ${ }^{60}$ ) would be quite small 64 . The genes underlying ecologically important phenotypes may instead be most useful for answering questions about the extent to which the genomic basis of traits is predictable and for identifying genetic constraints to adaptation, as developed in the next section.

\section{Box 3 here}

\section{How repeatable are eco-evolutionary dynamics?}

Although case studies of eco-evolutionary dynamics continue to accumulate ${ }^{8}$, an open question is whether these dynamics function in a repeatable manner through time or across space. In principle, repeatable eco-evolutionary dynamics require both the evolutionary shifts in phenotypes and the ecological consequences that stem from those shifts to be repeatable. As explained in the prior section, the direct utility of genomic data in studying the ecological consequences of phenotypic change is limited and thus we explore how genomic data may contribute to our understanding of the repeatability of evolution, both at the level of individual genetic polymorphisms and at higher levels, in the context of eco-evolutionary dynamics.

Genomic data allow researchers to ask if repeated eco-evolutionary dynamics rely on consistent genomic changes within each interacting species. An alternative scenario is one where repeated dynamics simply require consistent phenotypic changes that can be achieved through various genomic changes. If the former is true and the dynamic relies on a specific genetic variant, this implies that the extent of standing genetic variation, gene flow, or possibly the mutation rate will strongly dictate when eco-evolutionary dynamics occur (even more so if the dynamic relies on a haplotype with multiple linked variants). If, alternatively, an eco-evolutionary dynamic can occur through various genomic changes, then repeated eco-evolutionary dynamics might be expected in small, genetically depauperate populations as well as their larger counterparts. Thus, by identifying the genomic basis of traits involved in eco-evolutionary changes, we gain insight into whether these dynamics are robust or dependent on evolutionary contingency.

Although cases of repeated phenotypic evolution have been used as model systems to study eco-evolutionary dynamics $22,24,25,68-70$, we currently have little data on the tendency of eco-evolutionary dynamics to involve repeated evolutionary changes at the 
same loci. So far, the only genomic information obtained during a complete ecoevolutionary feedback loop comes from Becks et al. ${ }^{4}$ who, studying a rotifer-algae system, found that evolutionary shifts in a defense trait were associated with expression changes in completely different sets of genes in two consecutive predator-prey cycles in a replicated chemostat environment. This suggests that multiple bouts of experimentally controlled selection pressure resulted in similar phenotypic change through completely different genomic mechanisms. The extent of parallelism at the gene level may depend on whether adaptation occurs from de novo mutation (low parallelism ${ }^{71-74}$ ) or shared standing genetic variation (greater parallelism ${ }^{74,75}$ ). At the level of a whole gene or at higher functional categories, parallelism is expected to be more common 71,74,76,77. Eco-evolutionary experiments that assess parallelism at a range of genomic scales, from the SNP to the pathway level, would be tremendously useful in providing genomic insights on the potential repeatability of eco-evolutionary dynamics.

If eco-evolutionary dynamics were found to rely on the presence of specific genetic variants or the co-occurrence of multiple genetic variants in linkage disequilibrium in the focal population, it would be tempting to conclude that these dynamics are fragile or contingent upon evolutionary history. Yet, this is not necessarily correct. For example, the required genetic variants may be of ancient origin in the evolving population (Box 1) and maintained through balancing selection. In other cases, the selectively favored variants may be fixed in individual populations with variation maintained across populations or even species and be "re-suspended" through gene flow within the broader population or species network 78,79 , leading to repeated eco-evolutionary dynamics when ecological conditions are similar. Both of these scenarios are testable with population genomic techniques and demographic modeling. On the whole, genomic data could provide a metric of the repeatability of the evolutionary component of ecoevolutionary dynamics, insight into the origin of genomic variants that underlie variation in salient phenotypes, and a better resolved mechanistic basis for ecoevolutionary change each of which would advance our understanding of the repeatability of eco-evolutionary dynamics.

\section{Conclusion}

Although our understanding of eco-evolutionary dynamics continues to progress, the field remains dominated by review papers and a small number of well-resolved examples. Building a predictive understanding of when and how eco-evolutionary mechanisms operate in nature is still a distant goal. Clearly, genomic data provide only part of what is necessary to advance this understanding, and a number of the most powerful inferences that can made from genomic data are currently constrained to a 
small subset of nature. Major advances in our understanding of eco-evolutionary dynamics will still rely on experiments and observational data.

Nonetheless, given the central role of rapid evolution in driving eco-evolutionary dynamics, a true mechanistic understanding of these dynamics will likely require genomic data. As we have argued here, genomic tools can play key roles in efforts to evaluate the rate of evolutionary change, the traits under selection, the evolutionary history of relevant trait variation, and the genomic basis of these traits. These tools thereby help reveal the natural history of rapid evolutionary change in ecologically important species traits. Only once this natural history is better resolved across a wide range of systems will ecologists and evolutionary biologists be best positioned to advance a predictive understanding of eco-evolutionary dynamics.

\section{Box 1: When the new is old: the importance of old haplotypes in driving rapid evolution}

Although evolution operating on contemporary or rapid timescales ${ }^{8}$ is foundational to eco-evolutionary dynamics, in some key examples of this process, the genetic variation changing in frequency is quite old. For example, variation at a major effect haplotype for beak size in Darwin's finches is associated with differential survival and rapid trait evolution in the medium ground finch. However, this variation dates to early in the finch radiation and has repeatedly been introduced to new populations and species through hybridization ${ }^{38}$. Similarly, haplotype variation associated with freshwater adaptation in threespine stickleback fish likely long pre-dates the time when most extant populations colonized freshwater 12,80. Furthermore, haplotype variation associated with adaptation to different light regimes and rapid speciation in Lake Victoria cichlid fish has been generated by hybridization between two ancient lineages 78. Hybridization can also play a role in generating the haplotypes that prime populations for rapid evolution (and hence eco-evolutionary dynamics) by bringing together new suites of allopatrically evolved alleles at interacting loci. Recombination can then link these adaptive alleles ${ }^{47}$. These insights into the origin of the genetic variation underlying rapid evolution and eco-evolutionary dynamics are only possible with genomic data and worthy of future study.

\section{Box 2: Using genomics to estimate heritability}

Many species that have prominent ecological roles may not be amenable to classic breeding experiments or observational parentage studies that allow for the estimation of the heritability of key traits. However, advances in methods that estimate the 
relatedness between individuals based on genomic data mean that measuring traits and collecting genome wide single nucleotide polymorphisms (SNP) data can now yield a reliable estimate of heritability for a given trait in many species $43,45,81$. Specifically, these approaches estimate the heritability by using thousands of markers to produce an estimate of relatedness between individuals (or genetic relatedness matrix (GRM)) and then fitting this matrix to phenotypic data in a mixed model that also includes other potential sources of variation (e.g. environmental or time data) ${ }^{82}$. How well the GRM approximates a classical pedigree-based relationship matrix is dependent on the number of markers used, the population size, and genome size 43,83. GRM based estimates of heritability can be used to help predict population responses to ecological or environmental changes ${ }^{84}$ or in an eco-evolutionary context when paired with the Hairston et al. ${ }^{2}$ approach, which would be an advance for studies of eco-evolutionary dynamics in the wild.

\section{Box 3: Advances in sequencing technologies and bioinformatics: new data for eco- evolutionary dynamics}

Modern approaches based on reduced representation of the genome such as restriction site-associated DNA sequencing (RAD ${ }^{85}$ ), genotyping by sequencing (GBS ${ }^{86}$ ), multiplex shotgun genotyping 87 and exome capture and sequencing 88 allow for cost-effective genotyping of a large number of SNPs across multiple individuals. Current protocols for library preparation are easily transferred across systems and the number of markers obtained upon sequencing can be predicted conditional to the genome size (which can be estimated using flow cytometry ${ }^{89}$ or read depth in sequenced genomes ${ }^{90}$ and sequencing coverage ${ }^{91}$ ). Pool-seq ${ }^{92}$ and low-coverage sequencing of individually barcoded samples ${ }^{93,94}$ provide an increasingly affordable approach for more comprehensively screening the entire genome for variants associated with particular phenotypes or genes responding to selection pressures ${ }^{95}$. Sequencing technologies that produce drastically longer individual sequence reads, reducing the bioinformatic challenges and increasing the quality of the genome assembly considerably, have become more common ${ }^{96,97}$. These sequencing technologies, and other yet unknown advances, will greatly reduce the costs and effort associated with obtaining wellassembled genomes in non-model systems.

Bioinformatic approaches to make inferences from genomic data are likewise advancing at a tremendous rate. Bioinformatic processing of reduced representation genomic data does not require the availability of a reference genome, which can be replaced by a local de-novo assembly of reference contigs produced from the sequencing reads of samples. For example, bioinformatic processing of RAD data can make use of analytical tools developed for more general handling of genomic data, and can largely be customized by 
the operator. In addition, a number of comprehensive packages have been produced that allow the processing of RAD data with minimal knowledge of bioinformatics, and render the technique accessible to a broad audience of biologists ${ }^{98-100}$. Advances have also been made for those choosing to sequence whole genomes. Emerging approaches that utilize haplotype information from deep sequenced genetic lines could allow for accurate estimates of allele frequency when sequencing at low coverages in subsequent work. Advances in sequencing technologies are relevant to eco-evolutionary dynamics as they make it easier and cheaper to measure the pace of evolution (question 1), estimate heritabilities (Box 2), carry out association studies using genomic data (question 3), and provide the data for deeper questions about the evolutionary change that occurs in eco-evolutionary dynamics (Box 1 and question 4).

1. Yoshida, T., Jones, L. E., Ellner, S. P., Fussmann, G. F. \& Hairston, N. G. Rapid evolution drives ecological dynamics in a predator-prey system. Nature 424, 303-306 (2003).

2. Hairston, N. G., Ellner, S. P., Geber, M. A., Yoshida, T. \& Fox, J. A. Rapid evolution and the convergence of ecological and evolutionary time. Ecol. Lett. 8, 1114-1127 (2005).

3. Turcotte, M. M., Reznick, D. N. \& Hare, J. D. The impact of rapid evolution on population dynamics in the wild: experimental test of eco-evolutionary dynamics. Ecol. Lett. 14, 10841092 (2011).

4. Becks, L., Ellner, S. P., Jones, L. E. \& Hairston, N. G. The functional genomics of an ecoevolutionary feedback loop: linking gene expression, trait evolution, and community dynamics. Ecol. Lett. 15, 492-501 (2012).

5. Thompson, J. N. Rapid evolution as an ecological process. Trends Ecol. Evol. 13, 329-332 (1998).

6. Post, D. M. \& Palkovacs, E. P. Eco-evolutionary feedbacks in community and ecosystem ecology: interactions between the ecological theatre and the evolutionary play. Philos. Trans. R. Soc. Lond. B Biol. Sci. 364, 1629-1640 (2009). 
7. Schoener, T. W. The newest synthesis: understanding the interplay of evolutionary and ecological dynamics. Science 331, 426-429 (2011).

8. Hendry, A. P. Eco-Evolutionary Dynamics. (Princeton University Press, 2017).

9. Turcotte, M. M., Reznick, D. N. \& Daniel Hare, J. Experimental test of an eco-evolutionary dynamic feedback loop between evolution and population density in the green peach aphid. Am. Nat. 181 Suppl 1, S46-57 (2013).

10. Matthews, B., Aebischer, T., Sullam, K. E., Lundsgaard-Hansen, B. \& Seehausen, O. Experimental Evidence of an Eco-evolutionary Feedback during Adaptive Divergence. Curr. Biol. 26, 483-489 (2016).

11. Fussmann, G. F., Loreau, M. \& Abrams, P. A. Eco-evolutionary dynamics of communities and ecosystems. Funct. Ecol. 21, 465-477 (2007).

12. Jones, F. C. et al. The genomic basis of adaptive evolution in threespine sticklebacks. Nature 484, 55-61 (2012).

13. Savolainen, O., Lascoux, M. \& Merilä, J. Ecological genomics of local adaptation. Nat. Rev. Genet. 14, 807-820 (2013).

14. Seehausen, O. et al. Genomics and the origin of species. Nat. Rev. Genet. 15, 176-192 (2014).

15. Thurman, T. J. \& Barrett, R. D. H. The genetic consequences of selection in natural populations. Mol. Ecol. 25, 1429-1448 (2016).

16. Bergland, A. O., Behrman, E. L., O’Brien, K. R., Schmidt, P. S. \& Petrov, D. A. Genomic Evidence of Rapid and Stable Adaptive Oscillations over Seasonal Time Scales in Drosophila. PLoS Genet. 10, e1004775 (2014).

17. Stapley, J. et al. Adaptation genomics: the next generation. Trends Ecol. Evol. 25, 705-712 (2010).

18. Hendry, A. P. Key questions in the genetics and genomics of eco-evolutionary dynamics. Heredity 111, 456-466 (2013).

19. Slobodkin, L. B. Growth and regulation of animal populations. (Dover Publications., 1980).

20. Hendry, A. P. \& Kinnison, M. T. Perspective: The Pace of Modern Life: Measuring Rates of 
Contemporary Microevolution. Evolution 53, 1637-1653 (1999).

21. Alberti, M. et al. Global urban signatures of phenotypic change in animal and plant populations. Proc. Natl. Acad. Sci. U. S. A. (2017). doi:10.1073/pnas.1606034114

22. Post, D. M., Palkovacs, E. P., Schielke, E. G. \& Dodson, S. I. Intraspecific variation in a predator affects community structure and cascading trophic interactions. Ecology 89, 2019-2032 (2008).

23. Harmon, L. J. et al. Evolutionary diversification in stickleback affects ecosystem functioning. Nature 458, 1167-1170 (2009).

24. Bassar, R. D. et al. Local adaptation in Trinidadian guppies alters ecosystem processes. Proc. Natl. Acad. Sci. U. S. A. 107, 3616-3621 (2010).

25. Rudman, S. M. \& Schluter, D. Ecological Impacts of Reverse Speciation in Threespine Stickleback. Curr. Biol. 26, 490-495 (2016).

26. Endler, J. A. Natural Selection in the Wild. (Princeton University Press, 1986).

27. Reznick, D. N. \& Bryga, H. Life-History Evolution in Guppies (Poecilia reticulata): 1. Phenotypic and Genetic Changes in an Introduction Experiment. Evolution 41, 1370-1385 (1987).

28. Gienapp, P., Teplitsky, C., Alho, J. S., Mills, J. A. \& Merilä, J. Climate change and evolution: disentangling environmental and genetic responses. Mol. Ecol. 17, 167-178 (2008).

29. Vitti, J. J., Grossman, S. R. \& Sabeti, P. C. Detecting natural selection in genomic data. Annu. Rev. Genet. 47, 97-120 (2013).

30. Quesada, H., Ramírez, U. E. M., Rozas, J. \& Aguadé, M. Large-scale adaptive hitchhiking upon high recombination in Drosophila simulans. Genetics 165, 895-900 (2003).

31. Ohashi, J., Naka, I. \& Tsuchiya, N. The impact of natural selection on an ABCC11 SNP determining earwax type. Mol. Biol. Evol. 28, 849-857 (2011).

32. Gompert, Z. et al. Experimental evidence for ecological selection on genome variation in the wild. Ecol. Lett. 17, 369-379 (2014).

33. Kinnison, M. T., Hairston, N. G., Jr \& Hendry, A. P. Cryptic eco-evolutionary dynamics. Ann. N. 
Y. Acad. Sci. 1360, 120-144 (2015).

34. Excoffier, L., Hofer, T. \& Foll, M. Detecting loci under selection in a hierarchically structured population. Heredity 103, 285-298 (2009).

35. Bergland, A. O., Tobler, R., Gonzalez, J., Schmidt, P. \& Petrov, D. Secondary contact and local adaptation contribute to genome-wide patterns of clinal variation in Drosophila melanogaster. Mol. Ecol. (2016).

36. Nuzhdin, S. V. \& Turner, T. L. Promises and limitations of hitchhiking mapping. Curr. Opin. Genet. Dev. 23, 694-699 (2013).

37. Franssen, S. U., Nolte, V., Tobler, R. \& Schlötterer, C. Patterns of Linkage Disequilibrium and Long Range Hitchhiking in Evolving Experimental Drosophila melanogaster Populations. Mol. Biol. Evol. 32, 495-509 (2015).

38. Lamichhaney, S. et al. A beak size locus in Darwin's finches facilitated character displacement during a drought. Science 352, 470-474 (2016).

39. Johnson, M. T. J. \& Stinchcombe, J. R. An emerging synthesis between community ecology and evolutionary biology. Trends Ecol. Evol. 22, 250-257 (2007).

40. Rudman, S. M. et al. Adaptive genetic variation mediates bottom-up and top-down control in an aquatic ecosystem. Proc. Biol. Sci. 282, 20151234 (2015).

41. Ellner, S. P., Geber, M. \& Hairston, N. Does rapid evolution matter? Measuring the rate of contemporary evolution and its impacts on ecological dynamics. Ecol. Lett. 14, 603-614 (2011).

42. Govaert, L., Pantel, J. H. \& De Meester, L. Eco-evolutionary partitioning metrics: assessing the importance of ecological and evolutionary contributions to population and community change. Ecol. Lett. 19, 839-853 (2016).

43. Stanton-Geddes, J., Yoder, J. B., Briskine, R., Young, N. D. \& Tiffin, P. Estimating heritability using genomic data. Methods Ecol. Evol. 4, 1151-1158 (2013).

44. Bérénos, C., Ellis, P. A., Pilkington, J. G. \& Pemberton, J. M. Estimating quantitative genetic parameters in wild populations: a comparison of pedigree and genomic approaches. Mol. 
Ecol. 23, 3434-3451 (2014).

45. Visscher, P. M. et al. Assumption-Free Estimation of Heritability from Genome-Wide Identity-by-Descent Sharing between Full Siblings. PLoS Genet. 2, e41 (2006).

46. Pelletier, F. et al. Eco-evolutionary dynamics in a contemporary human population. Nat. Commun. 8, 15947 (2017).

47. Becks, L., Ellner, S. P., Jones, L. E. \& Hairston, N. G., Jr. Reduction of adaptive genetic diversity radically alters eco-evolutionary community dynamics. Ecol. Lett. 13, 989-997 (2010).

48. Agrawal, A. A., Hastings, A. P., Johnson, M. T. J., Maron, J. L. \& Salminen, J.-P. Insect herbivores drive real-time ecological and evolutionary change in plant populations. Science 338, 113-116 (2012).

49. Agrawal, A. A., Johnson, M. T. J., Hastings, A. P. \& Maron, J. L. A field experiment demonstrating plant life-history evolution and its eco-evolutionary feedback to seed predator populations. Am. Nat. 181 , S35-45 (2013).

50. McGill, B. J., Enquist, B. J., Weiher, E. \& Westoby, M. Rebuilding community ecology from functional traits. Trends Ecol. Evol. 21, 178-185 (2006).

51. Drysdale, R. \& FlyBase Consortium. FlyBase : a database for the Drosophila research community. Methods Mol. Biol. 420, 45-59 (2008).

52. Berardini, T. Z. et al. The Arabidopsis information resource: Making and mining the 'gold standard' annotated reference plant genome. Genesis 53, 474-485 (2015).

53. Ran, F. A. et al. Genome engineering using the CRISPR-Cas9 system. Nat. Protoc. 8, 22812308 (2013).

54. Shen, L., Courtois, B., McNally, K. L., Robin, S. \& Li, Z. Evaluation of near-isogenic lines of rice introgressed with QTLs for root depth through marker-aided selection. Theor. Appl. Genet. 103, 75-83 (2001).

55. Alonso, J. M. et al. Genome-wide insertional mutagenesis of Arabidopsis thaliana. Science 301, 653-657 (2003). 
56. Giaever, G. \& Nislow, C. The yeast deletion collection: a decade of functional genomics. Genetics 197, 451-465 (2014).

57. Arnegard, M. E. et al. Genetics of ecological divergence during speciation. Nature 511, 307311 (2014).

58. McKown, A. D. et al. Genome-wide association implicates numerous genes underlying ecological trait variation in natural populations of Populus trichocarpa. New Phytol. 203, 535-553 (2014).

59. McCarthy, M. I. et al. Genome-wide association studies for complex traits: consensus, uncertainty and challenges. Nat. Rev. Genet. 9, 356-369 (2008).

60. Whitham, T. G. et al. Community and ecosystem genetics: A consequence of the extended phenotype. Ecology 84, 559-573 (2003).

61. Bailey, J. K. et al. From genes to ecosystems: a synthesis of the effects of plant genetic factors across levels of organization. Philos. Trans. R. Soc. Lond. B Biol. Sci. 364, 1607-1616 (2009).

62. Schweitzer, J. et al. Genetically based trait in a dominant tree affects ecosystem processes. Ecol. Lett. 7, 127-134 (2004).

63. Hanski, I. \& Saccheri, I. Molecular-level variation affects population growth in a butterfly metapopulation. PLoS Biol. 4, e129 (2006).

64. Crutsinger, G. M. et al. Testing a 'genes-to-ecosystems' approach to understanding aquaticterrestrial linkages. Mol. Ecol. 23, 5888-5903 (2014).

65. Mackay, T. F. C., Stone, E. A. \& Ayroles, J. F. The genetics of quantitative traits: challenges and prospects. Nat. Rev. Genet. 10, 565-577 (2009).

66. Rockman, M. V. The QTN program and the alleles that matter for evolution: all that's gold does not glitter. Evolution 66, 1-17 (2012).

67. Barbour, M. A. et al. Multiple plant traits shape the genetic basis of herbivore community assembly. Funct. Ecol. 29, 995-1006 (2015).

68. Farkas, T. E., Mononen, T., Comeault, A. A., Hanski, I. \& Nosil, P. Evolution of camouflage 
drives rapid ecological change in an insect community. Curr. Biol. 23, 1835-1843 (2013).

69. Hiltunen, T. \& Becks, L. Consumer co-evolution as an important component of the ecoevolutionary feedback. Nat. Commun. 5, 5226 (2014).

70. Alexander, T. J., Vonlanthen, P. \& Seehausen, O. Does eutrophication-driven evolution change aquatic ecosystems? Philos. Trans. R. Soc. Lond. B Biol. Sci. 372, (2017).

71. Tenaillon, O. et al. The molecular diversity of adaptive convergence. Science 335, 457-461 (2012).

72. Lang, G. I. et al. Pervasive genetic hitchhiking and clonal interference in forty evolving yeast populations. Nature 500, 571-574 (2013).

73. Lind, P. A., Farr, A. D., Rainey, P. B. \& Shou, W. Experimental evolution reveals hidden diversity in evolutionary pathways. eLife Sciences 4, e07074 (2015).

74. Long, A., Liti, G., Luptak, A. \& Tenaillon, O. Elucidating the molecular architecture of adaptation via evolve and resequence experiments. Nat. Rev. Genet. 16, 567-582 (2015).

75. Burke, M. K. et al. Genome-wide analysis of a long-term evolution experiment with Drosophila. Nature 467, 587-590 (2010).

76. Renaut, S., Owens, G. L. \& Rieseberg, L. H. Shared selective pressure and local genomic landscape lead to repeatable patterns of genomic divergence in sunflowers. Mol. Ecol. 23, $311-324$ (2014).

77. Telonis-Scott, M., Sgrò, C. M., Hoffmann, A. A. \& Griffin, P. C. Cross-Study Comparison Reveals Common Genomic, Network, and Functional Signatures of Desiccation Resistance in Drosophila melanogaster. Mol. Biol. Evol. 33, 1053-1067 (2016).

78. Meier, J. I. et al. Ancient hybridization fuels rapid cichlid fish adaptive radiations. Nat. Commun. 8, 14363 (2017).

79. Ralph, P. L. \& Coop, G. The Role of Standing Variation in Geographic Convergent Adaptation. Am. Nat. 186, S5-23 (2015).

80. Colosimo, P. F. et al. Widespread Parallel Evolution in Sticklebacks by Repeated Fixation of Ectodysplasin Alleles. Science 307, 1928-1933 (2005). 
81. Yang, J., Lee, S. H., Goddard, M. E. \& Visscher, P. M. GCTA: a tool for genome-wide complex trait analysis. Am. J. Hum. Genet. 88, 76-82 (2011).

82. Kruuk, L. E. B. Estimating genetic parameters in natural populations using the 'animal model'. Philos. Trans. R. Soc. Lond. B Biol. Sci. 359, 873-890 (2004).

83. Wang, J. Pedigrees or markers: Which are better in estimating relatedness and inbreeding coefficient? Theor. Popul. Biol. 107, 4-13 (2016).

84. Gienapp, P. et al. Predicting demographically sustainable rates of adaptation: can great tit breeding time keep pace with climate change? Philos. Trans. R. Soc. Lond. B Biol. Sci. 368, 20120289 (2013).

85. Baird, N. A. et al. Rapid SNP Discovery and Genetic Mapping Using Sequenced RAD Markers. PLoS One 3, e3376 (2008).

86. Elshire, R. J. et al. A Robust, Simple Genotyping-by-Sequencing (GBS) Approach for High Diversity Species. PLoS One 6, e19379 (2011).

87. Andolfatto, P. et al. Multiplexed shotgun genotyping for rapid and efficient genetic mapping. Genome Res. 21, 610-617 (2011).

88. Bamshad, M. J. et al. Exome sequencing as a tool for Mendelian disease gene discovery. Nat. Rev. Genet. 12, 745-755 (2011).

89. Dolezel, J. \& Bartos, J. Plant DNA flow cytometry and estimation of nuclear genome size. Ann. Bot. 95, 99-110 (2005).

90. Li, R. et al. The sequence and de novo assembly of the giant panda genome. Nature 463, 311-317 (2010).

91. Mora-Márquez, F., García-Olivares, V., Emerson, B. C. \& López de Heredia, U. ddradseqtools: a software package for in silico simulation and testing of double-digest RADseq experiments. Mol. Ecol. Resour. 17, 230-246 (2017).

92. Schlötterer, C., Tobler, R., Kofler, R. \& Nolte, V. Sequencing pools of individuals - mining genome-wide polymorphism data without big funding. Nat. Rev. Genet. 15, 749-763 (2014). 
93. Therkildsen, N. O. \& Palumbi, S. R. Practical low-coverage genomewide sequencing of hundreds of individually barcoded samples for population and evolutionary genomics in nonmodel species. Mol. Ecol. Resour. 17, 194-208 (2017).

94. Alex Buerkle, C. \& Gompert, Z. Population genomics based on low coverage sequencing: how low should we go? Mol. Ecol. 22, 3028-3035 (2013).

95. Lowry, D. B. et al. Breaking RAD: an evaluation of the utility of restriction site-associated DNA sequencing for genome scans of adaptation. Mol. Ecol. Resour. 17, 142-152 (2017).

96. Mostovoy, Y. et al. A hybrid approach for de novo human genome sequence assembly and phasing. Nat. Methods 13, 587-590 (2016).

97. Dudchenko, O. et al. De novo assembly of the Aedes aegypti genome using Hi-C yields chromosome-length scaffolds. Science 356, 92-95 (2017).

98. Catchen, J., Hohenlohe, P. A., Bassham, S., Amores, A. \& Cresko, W. A. Stacks: an analysis tool set for population genomics. Mol. Ecol. 22, 3124-3140 (2013).

99. Eaton, D. A. R. PyRAD: assembly of de novo RADseq loci for phylogenetic analyses. Bioinformatics 30, 1844-1849 (2014).

100. Puritz, J. B., Hollenbeck, C. M. \& Gold, J. R. dDocent: a RADseq, variant-calling pipeline designed for population genomics of non-model organisms. PeerJ 2, e431 (2014).

Please address correspondence to Seth Rudman (srudman@sas.upenn.edu).

\section{Acknowledgements}

The paper was conceived during a Monte Verita conference on 'The Genomic Basis of Eco-Evolutionary Change' organized by the Centre for Adaptation to a Changing Environment (ACE) at ETH Zürich. We thank the Congressi Stefano Franscini and ETH Zürich for funding and supporting the meeting.

\section{Authorship statement}

SMR assembled the first draft of the manuscript based on contributions from all authors. All authors provided revisions. 
Figure 1: Five major questions in eco-evolutionary dynamics

A schematic showing the reciprocal interactions between genomic evolution, phenotypic evolution, and ecological dynamics. An eco-evolutionary feedback loop occurs when genomic evolution drives phenotypic evolution, which in turn influences ecological dynamics that feedback to affect phenotypic and genomic evolution. The five questions organizing this paper are displayed in relation to the causal arrow or arrows they investigate.

Figure 2: Using genomic tools to study a predator-prey eco-evolutionary dynamic Trait evolution (upper panel) and its population dynamics consequences (lower panel) through time. Box arrows indicate the eco-evolutionary information that can be obtained from sequencing at a single time point (estimate trait heritability with GRM (see Box 2) and measure the community level impacts of large effect genes), sequencing through time (measure the pace of evolution, use of genotype-phenotype linkages to find evolving traits) and combining sequence and population dynamics data for two species interacting over time (measure eco-evolutionary dynamics between two species (at the genetic level)). 